Validity and reliability of an ultrasound measurement of the free length of the Achilles tendon

Barfod, Kristoffer Weisskirchner; Riecke, Anja Falk; Boesen, Anders; Hansen, Philip; Maier, Jens Friedrich; Doessing, Simon; Troelsen, Anders

Published in:

Danish Medical Journal

Publication date:

2018

Document version

Publisher's PDF, also known as Version of record

Document license:

Unspecified

Citation for published version (APA):

Barfod, K. W., Riecke, A. F., Boesen, A., Hansen, P., Maier, J. F., Doessing, S., \& Troelsen, A. (2018). Validity and reliability of an ultrasound measurement of the free length of the Achilles tendon. Danish Medical Journal, 65(3), [A5453]. http://ugeskriftet.dk/dmj/validity-and-reliability-ultrasound-measurement-free-length-achillestendon 


\section{Validity and reliability of an ultrasound measurement of the free length of the Achilles tendon}

Kristoffer Weisskirchner Barfod ${ }^{1}$, Anja Falk Riecke ${ }^{2}$, Anders Boesen ${ }^{1}$, Philip Hansen $^{3}$, Jens Friedrich Maier ${ }^{4}$, Simon Doessing ${ }^{5}$ \& Anders Troelsen ${ }^{6}$

\begin{abstract}
INTRODUCTION: Valid length measurements of the different segments of the Achilles tendon are needed in order to investigate if differential elongation of the Achilles tendon takes place after rupture. The purpose of this paper was to present data concerning the accuracy and reliability of an ultrasound measurement of the free part of the Achilles tendon.
\end{abstract}

METHODS: Both legs of 19 non-injured subjects were examined by magnetic resonance imagining (MRI) and ultrasound. The length from the distal tip of the soleus muscle to the tendon insertion on the calcaneus was measured by three independent ultrasound examiners. Repeated ultrasound measurements were performed and compared with MRI measurements. Intra-rater and inter-rater reliability and the agreement between MRI and ultrasound were determined. Data were evaluated using the intraclass correlation coefficient (ICC), the standard error of the measurement (SEM) and the minimal detectable change (MDC). RESULTS: The measurement showed excellent intra-rater reliability (ICC $=0.94$ ( $95 \%$ confidence interval $(\mathrm{Cl})$ : 0.91$0.96), \mathrm{SEM}=5 \mathrm{~mm}$ and $\mathrm{MDC}=13 \mathrm{~mm}$ ) and inter-rater reliability (ICC $=0.96$ (95\% Cl: 0.93-0.97), SEM = $4 \mathrm{~mm}$ and $\mathrm{MDC}=11 \mathrm{~mm}$ ). On average, ultrasound measurements exceeded the MRI measurements by $2 \mathrm{~mm}$ (non-significant); resulting in a measurement error of $5 \%$.

CONCLUSIONS: The ultrasound measurement of the free part of the Achilles tendon showed good reliability and accuracy. For comparison between groups of non-injured subjects, differences of $>5 \mathrm{~mm}$ can be detected. For repeated assessment of individual subject differences $\geq 13 \mathrm{~mm}$ can be detected.

FUNDING: none.

TRIAL REGISTRATION: Institutional Review Board of Zealand, Denmark, Ref. no: SJ-318.

Achilles tendon rupture is a devastating injury causing many patients to suffer from chronically impaired locomotor function, and only $30-66 \%$ resume previous sports activities [1, 2]. The debilitating effect of such injury is suggested to be associated with elongation of the Achilles tendon, leaving the triceps surae muscle at an ineffective working length $[3,4]$. This leads to reduced plantar flexion force of the ankle, thereby impairing a powerful lift-off [5].

The Achilles tendon provides linkage between the calcaneus and the three muscle bellies of the triceps surae: the soleus, the medial and the lateral head of gastrocnemius $[6,7]$. It is often considered one tendon moving "en bloc" [5, 7], but studies suggest that the separate collagen fibre bundles can move independently of each other, allowing for differential displacement of the three parts of the tendon during normal muscle contraction $[6,8,9]$. If this is the case, the three muscle bellies might retract differently after rupture due to differentiated elongation of the three parts of the tendon. The medial head of the gastrocnemius muscle shows the greatest amount of contraction during normal locomotion [8]. It might therefore be expected that this part of the tendon elongates the most after rupture. A differentiated elongation of the Achilles tendon might result in altered walking and running patterns due to alterations in force transmission.

Valid length measurements of the different parts of the tendon are needed to investigate if rupture causes differential elongation of the Achilles tendon. An ultrasound (US)-based measurement of the length from the calcaneus to the medial head of the gastrocnemius muscle has previously been investigated at our clinic (Figure 1) [10] and showed excellent validity and reliability. This study also included measurements of the length from the calcaneus to the distal tip of the soleus muscle (Figure 1), but those data were not included in the paper as the Achilles tendon was thought to be moving "en bloc". The aim of the paper was to develop a measurement that could be used in the acute phase of rupture, and the distal tip of the soleus was thought to be an unreliable anatomical landmark in the acute phase of rupture due to the proximity of the rupture haematoma.

The purpose of the present paper is to present data concerning the accuracy and reliability of a clinically applicable and easy to perform US measurement of the free part of the Achilles tendon (Figure 1).

\section{METHODS}

The study was performed in the same setting as previously described for the development and validation of an US measure from the calcaneus to the most distal tip of the medial gastrocnemius head [10]. Measurements were performed on the same subjects by the same investigators. The study population was first scanned by

\section{ORIGINAL \\ ARTICLE}

1) Department of Orthopaedics, Sports Orthopaedic Research Centre - Copenhagen (SORC-C), Hvidovre Hospital 2) Department of Orthopaedics, Zealand University Hospital Køge

3) Department of Radiology, Bispebjerg \& Frederiksberg Hospital

4) Department of Radiology, Zealand University Hospital Køge

5) Institute of Sports Medicine, Bispebjerg \& Frederiksberg Hospital 6) Department of Orthopaedics, Clinical Orthopaedic Research Hvidovre, Hvidovre Hospital, Denmark

Dan Med J 2018;65(3):A5453 


\section{FIGURE 1}

Panoramic ultrasound picture showing the posterior part of the calf. The distance from the calcaneus to the medial head of the gastrocnemius muscle (A). The free part of the Achilles tendon (B). The calcaneus (1), the soleus muscle (2), the medial head of the gatrocnemius muscle (3), the posterior-superior corner of the calcaneus (4), the distal tip of the soleus muscle (5), and the distal tip of the medial head of the gastrocnemius muscle (6).

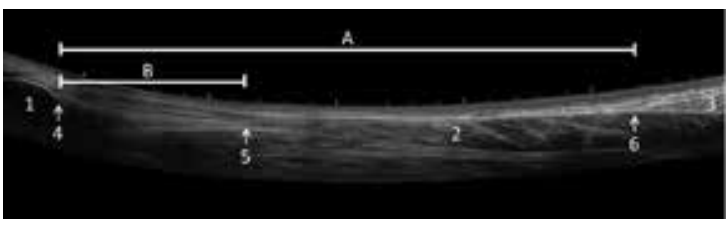

$\leftarrow \quad$ FIGURE 2

Positioning of the study subjects. Participants were positioned in a prone position with the knee flexed at $10-20^{\circ}$. The ankles rested on a triangular foam pad. A. Using a goniometer, the ankle joint was positioned in $10^{\circ}$ of plantar flexion by adjustment of the foam pad. B. With a marker, the point where the ultrasound probe and the needle crossed was marked on the skin. $\mathbf{C}$. The distance between landmarks was measured with a tape measure following the curves of the leg.

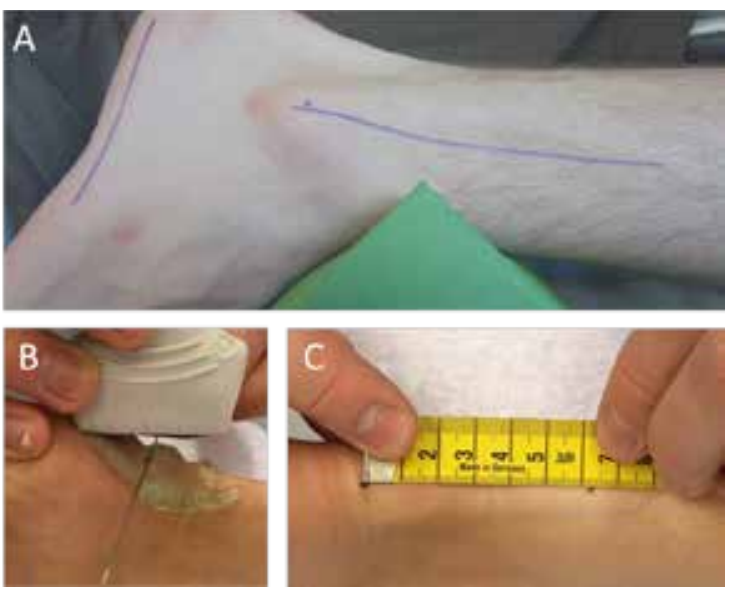

\section{FIGURE 3}

Sagittal ultrasound pictures showing the landmarks. A. The distal landmark is the posterior-superior corner of the calcaneus (1), the insertion of the Achilles tendon at the calcaneus (2), the Achilles tendon (3), the posterior acoustic shadowing of a 21-gauge needle projecting the landmark to the skin (4). B. The proximal landmark is the most distal muscle fibres' insertion into the Achilles tendon at the distal tip of the soleus muscle (5), the soleus muscle (6).
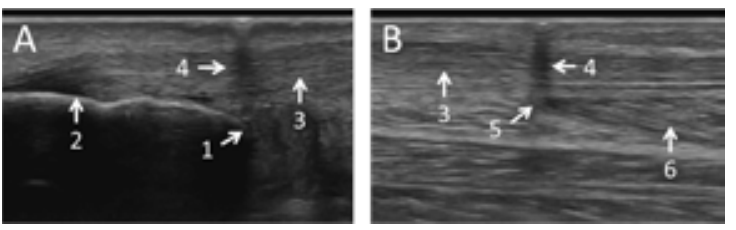

three independent US investigators; this was followed by MRI scans that were used as the gold standard to test concurrent validity.

\section{Description of the ultrasound measurement}

The length of the free part of the Achilles tendon was defined as the distance between the most proximal point of the posterior border of the calcaneus and the distal tip of the soleus muscle-tendon junction. The measurement was carried out in two steps; first the anatomical landmarks were identified and marked on the skin, and then the distance between them was measured on the skin with a tape measure [10]:

1) Positioning: Participants were positioned in a prone position with the knee flexed $10^{\circ}$. The feet rested on a triangular-shaped foam pad as shown in Figure 2A. Using a goniometer, the ankle joint was positioned in $10^{\circ}$ of plantar flexion by adjusting the foam pad.

2) Identification of the distal landmark: The distal landmark was defined as the posterior and most superior corner of the calcaneus in the midline, which was identified as the point where the cortical bone and its underlying acoustic shadow ended on sagittal US examination (Figure 3). The landmark was identified and centred in the image with the probe oriented in the sagittal plane. A 21-gauge needle was then introduced between the probe and the surface of the skin projecting the landmark to the skin. The projected point was marked on the skin with a non-permanent marker (Figure 2B).

3) Identification of the proximal landmark: The proximal landmark was defined as the distal tip of the soleus muscle. On US, this was defined as the point where the most distal muscular fibres inserted into the Achilles tendon (Figure 3). The landmark was identified, projected to the skin and marked in the same way as for the distal landmark.

4) Length measurement: The direct distance between landmarks was measured with a tape measure (accuracy $=1 \mathrm{~mm}$ ) along the contour of the skin (Figure 2).

\section{Study population}

The study was performed on the same nineteen noninjured subjects who participated in the previous study [10]. The subjects were aged 26-63 years and had no prior Achilles tendon problems. Investigations were performed in the period November 2012 to January 2013. All participants received oral and written information and their written consent was obtained. The study was approved by the Institutional Review Board of Zealand, Denmark, Ref. no: SJ-318. 


\section{Evaluation protocol}

The novel US measurement was evaluated by three independent US investigators with 2-5 years of experience within musculoskeletal US. Three different US scanners were used: GE Healthcare Logiq S8, Logiq 9 and logiq P5. The frequency was set to $15 \mathrm{MHz}$ and the focus was dynamically adjusted by the US operator. After each scan, the marks on the skin were removed in order to secure blinding of results between investigators.

Inter-rater and intra-rater reliability and agreement for the novel US measurement were determined for the three independent US investigators. Repeated measurements were performed at a three-week interval between scans. Subsequently, MRI examinations were performed within two months. MR images were evaluated by two independent investigators, both specialised in musculoskeletal MRI. All investigators were blinded to the results of the other investigators and to their own previous results. Participants were positioned identically for US and MRI investigations using the same triangleshaped foam pad. The position of the ankle and knee joint was controlled using a goniometer.

\section{Magnetic resonance imaging setup}

MR imaging was performed on a 1.5T system (Magnetom Avanto, Siemens, Erlangen, Germany). Axial 2D sequences covering both entire lower legs were performed as follows: T1-weighted without fat saturation (TR/TE, 485/12 ms; flip angle, $90^{\circ}$; pixel matrix, $384 \times 256$; voxel size, $1.2 \times 0.9 \times 6.0 \mathrm{~mm}$; slice thickness, $6.0 \mathrm{~mm}$; distance factor, $30 \%$; field of view (FOV), $350 \times 312 \mathrm{~mm}$; slices, 58) and T2-weighted without fat saturation (TR/ $\mathrm{TE}, 4,090 / 78 \mathrm{~ms}$; flip angle, $150^{\circ}$; pixel matrix, $448 \times$ 355 ; voxel size, $1.0 \times 0.8 \times 6.0 \mathrm{~mm}$; slice thickness, 6.0 $\mathrm{mm}$; distance factor, $20 \%$; FOV, $370 \times 367 \mathrm{~mm}$; slices, $60)$. Sagittal $2 \mathrm{D}$ sequences oriented in a plane perpendicular to the axis of the talocrural joint covering each entire lower leg separately were performed as follows: T1-weighted without fat saturation (TR/TE, 555/22 ms; flip angle, $180^{\circ}$; pixel matrix, $448 \times 238$; voxel size, 1.2 $\times 0.9 \times 3.0 \mathrm{~mm}$; slice thickness, $3.0 \mathrm{~mm}$; distance factor, 10\%; FOV, $410 \times 291 \mathrm{~mm}$; slices, 37) and T2-weighted without fat saturation (TR/TE, 4740/72 ms; flip angle, $150^{\circ}$; pixel matrix, $512 \times 512$; voxel size, $1.7 \times 1.7 \times 4.0$ $\mathrm{mm}$; slice thickness, $4.0 \mathrm{~mm}$; distance factor, $20 \%$; FOV, $440 \times 440 \mathrm{~mm}$; slices, 26).

T1-weighted slices were used for all measurements. Length measures were performed on the slice that showed the distal tip of the soleus muscle, covering the distance from the distal tip of the soleus muscle to the distal end of the Achilles tendon, which was defined by the axial plane intersecting the most cranial aspect of the tuber calcanei.

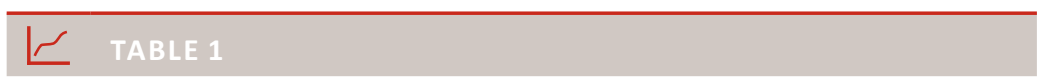

Results.

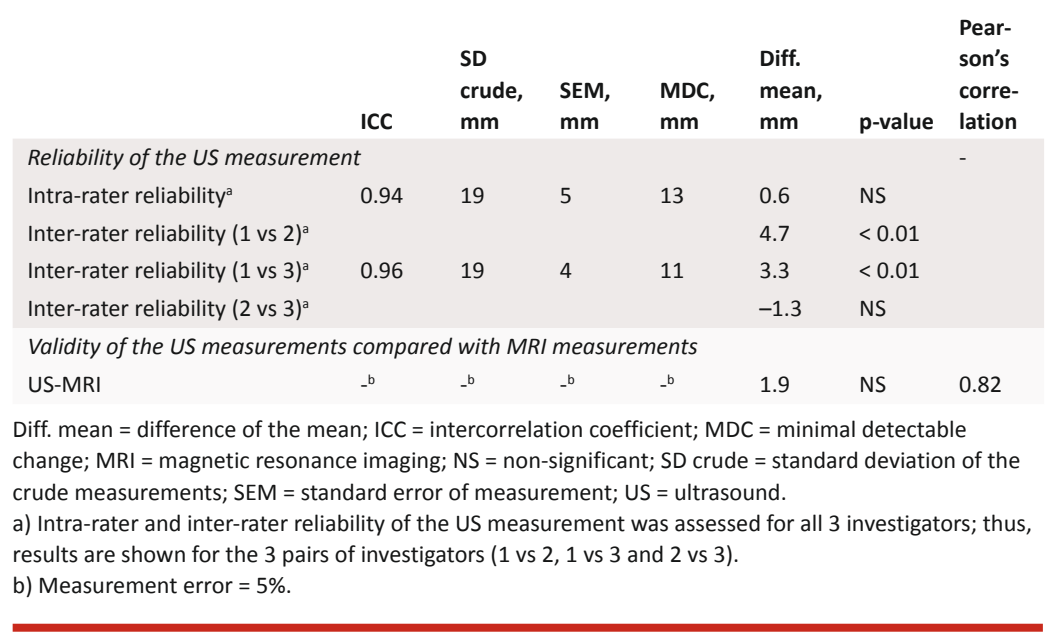

\section{Statistical methods}

In this exploratory study we a priori decided to obtain a sample size of 19 test persons using both legs. Standard procedures were used for descriptive statistics. Reliability was determined using the intercorrelation coefficient (ICC). ICC2.2 Two-way random, absolute agreement was used for evaluation of intra-rater reliability, whereas ICC2.3 Two-way random, absolute agreement was used for inter-rater reliability. Differences of the mean and standard error of the measurement (SEM) = standard deviation $(S D) \times V(1-I C C)$ were calculated to assess the agreement between groups of data [11]. The minimal detectable change (MDC) was calculated to assess agreement between data on the individual patients $(\mathrm{MDC}=1.96 \times \sqrt{ } 2 \times \mathrm{SEM})$ [11]. A paired t-test was used for comparison of means as the data showed a normal distribution and the scale was considered to be continuous. Correlation between US and MRI was assessed using Pearson's correlation. Statistical analyses were performed using the Statistical Package for Social Sciences (SPSS, version 22.0 for Windows, SPSS Inc., Chicago, III).

Trial registration: Institutional Review Board of Zealand, Denmark, Ref. no: SJ-318.

\section{RESULTS}

Both legs of 19 uninjured persons (eight males and 11 females) were studied. The mean age was 43.4 years (SD = 10.7, range: $26-63$ years). The average height was 175 $\mathrm{cm}(\mathrm{SD}=9$, range: $158-192 \mathrm{~cm}$ ) and the average weight was $76.8 \mathrm{~kg}$ (SD = 12.9, range: $58-110 \mathrm{~kg}$ ). All participants stated their right leg to be dominant. 


\section{Reliability}

The US measurement of the free part of the Achilles tendon showed excellent intra-rater reliability, good agreement (ICC = 0.94 (95\% confidence interval (CI): 0.91-0.96), $\mathrm{SEM}=5 \mathrm{~mm}$ and $\mathrm{MDC}=13 \mathrm{~mm}$ ) and good inter-rater reliability (ICC $=0.96$ (95\% Cl: 0.93-0.97), SEM = $4 \mathrm{~mm}$ and MDC = $11 \mathrm{~mm}$ ) (Table 1). There was no systematic difference between test days, but a systematic difference between investigators of up to $5 \mathrm{~mm}(p<0.01)$ was seen.

\section{Validity}

Validity of the US measurement was tested by comparison with the MRI measurements. The measurements showed good correlation $r=0.82$ according to the recommendations of Portney \& Watkins [12]. MRI measurements were, on average, $2 \mathrm{~mm}$ shorter than the US measurements (non-significant). The average length of the measured Achilles tendons was $42 \mathrm{~mm}$; resulting in a measurement error of $2 / 42=5 \%$.

\section{DISCUSSION}

The most important finding of the present study was that the US measurement of the free part of the Achilles tendon showed good reliability and accuracy in non-injured people. For comparison between groups of noninjured people, differences of $5 \mathrm{~mm}$ can be detected; and for repeated assessment of individual subjects, differences of $\geq 13 \mathrm{~mm}$ can be detected. In comparison with MRI, the US measurement showed an acceptable accuracy with a measurement error of $5 \%$.

Both the inter-rater and intra-rater reliability were fully comparable with measurements using extended field of view (EFOV) US imaging [13]. The advantage of EFOV measurement is its ease and the possibility of saving the pictures for later analysis and/or control. On the other hand, the advantage of the present US measurement is its relative ease and that it is a low-cost technology, making it applicable in any US setting.

The clinical relevance of the US measurement depends on its ability to detect elongations of the tendon. In relation to the average length of the free tendon (42 $\mathrm{mm}$ ), an elongation of $12 \%$ can be detected when comparing groups of patients, whereas the elongation must exceed $31 \%$ when assessing individual subjects. If the average elongation after rupture is $2.5-3.5 \mathrm{~cm}$, as described by Silbernagel et al [4], the US measure is fully capable of measuring differences in groups of patients, whereas one should take care in using it for assessment of individual patients.

When using the US measurement, one should be familiar with the anatomical landmarks and be aware not to move the skin while measuring. Our setup was strictly standardised in order to minimise the risk of such measurements errors.
When interpreting the results, it is important to consider the morphology of the distal tip of the soleus, which might change after an Achilles tendon rupture. Therefore, the present findings might not be directly transferable to a population with ruptured Achilles tendons. Particularly, care should be taken in the months following a rupture where the authors' experience is that the morphology of the distal tip of the soleus muscle is blurred and difficult to identify. Probably a measurement of the free part of the Achilles tendon, using the distal tip of the soleus muscle as a landmark, should not be performed until six months after injury in order for the landmark to become clear and easily identifiable. This needs to be investigated in a sample of patients with Achilles tendon rupture. This study is limited by the lack of a proper sample size calculation. The sample size was determined due to logistic matters and no power calculation was performed. Also, the standardised setup for the measurement might have put the tendon on slack, why the measurement is likely to underestimate the true length of the tendon.

\section{CONCLUSIONS}

The US measurement of the free part of the Achilles tendon showed good reliability and accuracy. For comparison between groups of non-injured subjects, differences of $>5 \mathrm{~mm}$ can be detected. For repeated assessment of individual subjects, differences of $>13 \mathrm{~mm}$ can be detected. The US measurement is a promising clinical tool to be further assessed in the setting of acute Achilles tendon rupture.

CORRESPONDENCE: Kristoffer Weisskirchner Barfod.

E-mail: kbarfod@dadlnet.dk

ACCEPTED: 15 January 2018

CONFLICTS OF INTEREST: Disclosure forms provided by the authors are available with the full text of this article at www.danmedj.dk

LITERATURE

1. Metz R, Verleisdonk E-JMM, van der Heijden GJ-M-G et al. Acute Achilles tendon rupture: minimally invasive surgery versus nonoperative treatment with immediate full weightbearing - a randomized controlled trial. Am J Sports Med 2008;36:1688-94.

2. Möller M, Movin T, Granhed $\mathrm{H}$ et al. Acute rupture of tendo Achillis. A prospective, randomized study of comparision between surgical and nonsurgical treatment. J Bone Jt Surg 2001;83:843-8.

3. Kangas J, Pajala A, Ohtonen P et al. Achilles tendon elongation after rupture repair: a randomized comparison of 2 postoperative regimens. Am J Sports Med 2007;35:59-64.

4. Silbernagel KG, Steele R, Manal K. Deficits in heel-rise height and achilles tendon elongation occur in patients recovering from an Achilles tendon rupture. Am J Sports Med 2012;40:1564-71.

5. Barfod KW, Bencke J, Lauridsen HB et al. Nonoperative, dynamic treatment of acute Achilles tendon rupture: influence of early weightbearing on biomechanical properties of the plantar flexor muscle-tendon complex-a blinded, randomized, controlled trial. J Foot Ankle Surg 2015;54:220-6.

6. Bojsen-M $\varnothing$ ller J, Hansen P, Aagaard P et al. Differential displacement of the human soleus and medial gastrocnemius aponeuroses during isometric plantar flexor contractions in vivo. J Appl Physiol 2004;97:190814.

7. Theobald $P$, Benjamin M, Nokes $L$ et al. Review of the vascularisation of the human Achilles tendon. Injury 2005;36:1267-72.

8. Bojsen-Møller J, Magnusson SP. Heterogeneous loading of the human Achilles tendon in vivo. Exerc Sport Sci Rev 2015;43:190-7.

9. Haraldsson $\mathrm{BT}$, Aagaard P, Qvortrup K et al. Lateral force transmission between human tendon fascicles. Matrix Biol 2008;27:86-95. 
10. Barfod KW, Riecke AF, Boesen A et al. Validation of a novel ultrasound measurement of achilles tendon length. Knee Surgery, Sport Traumatol Arthrosc 2014;23:3398-406.

11. de Vet HCW, Terwee CB, Knol DL et al. When to use agreement versus reliability measures. J Clin Epidemiol 2006;59:1033-9.

12. Portney LG, Watkins MP. Foundations of clinical research: applications to Practice. New Jersey: Prentice Hall Health, 2000.

13. Silbernagel K G. Extended field of view ultrasound imaging to evaluate Achilles tendon length and thickness: a reliability and validity study. Musc Liga Tendons J 2016;6:104-10. 